\title{
A General Korovkin Result Under Generalized Convergence
}

\author{
PEDRO GARRANCHO
}

\begin{abstract}
In this paper, the classic result of Korovkin about the convergence of sequences of functions defined from sequences of linear operators is reformulated in terms of generalized convergence. This convergence extends some others given in the literature. The operator of the sequence fulfill a shape preserving property more general than the positivity. This property is related with certain extension of the notion of derivative. This extended derivative is precisely the object of the approximation process. The study is completed by analysing the conditions for the existence of an asymptotic formula, from which some interesting consequences are derived as a local version of the shape preserving property. Finally, as applications of the previous results, the author use the following notion of generalized convergence, an extension of Nörlund-Cesáro summability given by V. Loku and N. L. Braha in 2017. A way to transfer a notion of generalized convergence to approximation theory by means of linear operators is showed.
\end{abstract}

Keywords: Korovkin results, asymptotic condition, generalized convergence.

2010 Mathematics Subject Classification: 41A36, 41A28, 40D05.

\section{INTRODUCTION}

The following sequence of positive linear operators is studied in [2]:

$$
B_{n}^{\tau} f(t)=\sum_{k=0}^{n}\left(\begin{array}{l}
n \\
k
\end{array}\right) \tau(t)^{k}(1-\tau(t))^{n-k}\left(f \circ \tau^{-1}\right)(k / n), \quad f \in C[0,1], t \in[0,1],
$$

where $\tau$ is a function defined on $[0,1]$ infinitely differentiable, such that $\tau(0)=0, \tau(1)=1$ and $\tau^{\prime}(t)>0, t \in(0,1)$. The convergence of $B_{n}^{\tau} f$ towards $f$ can be analyzed by using the classical result of Korovkin [11], according to which it suffices to check it for these three test functions $1, t, t^{2}$, or other three, say $\psi_{0}, \psi_{1}, \psi_{2}$ that form a Tchebychev System. In particular, the choice $1, \tau, \tau^{2}$ is the more convenient for $B_{n}^{\tau}$.

Now, let $L_{n}$ be a slight modification of the previous sequence of positive linear operators, $L_{n} f(t)=\left(1+a_{n}\right) B_{n}^{\tau} f(t)$, where $a_{n}$ does not converge to 0 in the classical sense. The aforementioned result of Korovkin allows to conclude that the approximation process defined by $L_{n}$ is not convergent. That said, if $a_{n}$ is convergent in some other sense, a question arises whether the sequence would be convergent under this other notion. This is a motivation for a long list of papers where the so called Korovkin theory has been extended by considering new notions of convergence. We mention a few, restricting our attention to sequences of linear operator defined on spaces of real continuous functions on a compact interval.

In 1970, J. P. King and J. J. Swetits [10] studied the almost convergence, introduced by Lorentz in 1948 [14]. In 2002, A. D. Gadjiev and C. Orhan[6] proceeded analogously with statistical convergence, a now classic concept that was conceived by H. Fast in 1957 [5]. More recently, we may mention some papers by V. Karakaya and A. Karaisa in 2015 [9], where they considered weighted $\alpha \beta$-statistical convergence, T. Acar and S. A. Mohiuddine in 2016 [1] dealt with

Received: 22 February 2019; Accepted: 1 April 2019; Published Online: 3 April 2019

*Corresponding author: P. Garrancho; pgarran@ujaen.es

DOI: $10.33205 / \mathrm{cma} .530987$ 
statistical $(C, 1)(E, 1)$ summability, or finally, in 2016, D. Ali Karaisa, [8] worked with statistical $\left(N^{\gamma}, \alpha \beta\right)$ summability.

All the quoted papers dealt with positive linear operators and more importantly, for the proofs of their main results the same arguments of continuity and boundness were strongly used. Our main purpose with this work is to bring a sort of unification by proving a general qualitative Korovkin result, in such a way that this result can be applied whenever a concept of convergence is moved from mathematical analysis to Korovkin-type approximation theory. Moreover, we shall deal with a shape preserving property more general than the mere positivity, related to the preservation of the sign of certain generalized derivative.

This paper is organized as follows. In section 2, we will show some required notions and the notation will be set. In section 3, the qualitative Korovkin type result will be shown. Besides this, in the section 4, we will add the analysis of existence of the asymptotic condition by means of another Korovkin type result. In section 5, some consequences of the existence of an asymptotic condition will be given. In the last section, we show an example that shows the applicability of our result, by recovering the paper by V. Loku and N. L. Braha [12].

\section{GENERAL SETTINGS}

In this section, we will establish the framework, and present the required tools. Some notation will be set as well.

Let $\mathcal{S}$ be the usual linear space of all real sequences, and let $\mathcal{S}_{0}$ be a subspace of $\mathcal{S}$ closed under the usual sum and scalar multiplication.

Let $\mathcal{L}$ be a linear functional defined on $\mathcal{S}_{0}$ fulfilling the following properties:

(I) if $x_{n}$ is convergent in the classical sense, then $\mathcal{L}\left(x_{n}\right)=\lim x_{n}$, where lim refers to the classic limit (as a consequence $\mathcal{L}\left(x_{n}\right)=\ell \quad \Leftrightarrow \quad \mathcal{L}\left(x_{n}-\ell\right)=0$ );

(II) if $x_{n} \leq y_{n}$ for every $n \in \mathbb{N}$, then $\mathcal{L}\left(x_{n}\right) \leq \mathcal{L}\left(y_{n}\right)$ for every $n \in \mathbb{N}$. In short, $x_{n} \leq y_{n}$ implies $\mathcal{L}\left(x_{n}\right) \leq \mathcal{L}\left(y_{n}\right)$

(III) if $a_{n}$ is non negative, $\lim a_{n}=0$ and $\mathcal{L}\left(x_{n}\right)=\ell$, then $\mathcal{L}\left(a_{n} \cdot x_{n}\right)=0$;

(IV) if $x_{n} \leq z_{n} \leq y_{n}$ and $\mathcal{L}\left(x_{n}\right)=\mathcal{L}\left(y_{n}\right)=\ell$, then $z_{n} \in \mathcal{S}_{0}$ and $\mathcal{L}\left(z_{n}\right)=\ell$.

We have assumed, and will assume from now onwards that $x_{n} \in \mathcal{S}_{0}$ whenever we write $\mathcal{L}\left(x_{n}\right)$. On the other hand, to fix ideas, notice that under the classical setting $\mathcal{S}_{0}$ is formed by all convergent sequences, and, under statistical convergence, our functional $\mathcal{L}$ coincides with the so noted st - lim.

Recall the following properties for a Tschebyshev System, $T=\left\{\psi_{0}, \psi_{1}, \psi_{2}\right\}$, on an interval $[a, b]$ :

P1: Given three points $x_{1}, x_{2}, x_{3} \in[a, b]$ and three real numbers $a_{1}, a_{2}, a_{3}$, there exists only one $T$-polynomial (i.e. a function that belongs to the space spanned by $\psi_{0}, \psi_{1}, \psi_{2}$ ), such that $p_{T}\left(x_{i}\right)=a_{i}, \quad i=1,2,3$.

P2: For all $\alpha \in(a, b)$, we can find a $T$-polynomial, $p_{T, \alpha}$, such that, $\alpha$ is a double root of $p_{T, \alpha}$.

As it is usual $C^{m}[0,1]$ is the set of the all functions $m$-times differentiable with continuous $m$-th derivative. Notice that $C[0,1]$ is simply the set of continuous function on $[0,1]$ and $C^{\infty}[0,1]=$ $\cap_{i \in \mathbb{N}} C^{i}[0,1]$. Now let $\tau \in C^{\infty}[0,1]$, with $\tau(0)=0, \tau(1)=1$ and $\tau^{\prime}(t)>0, t \in(0,1)$. In relation with the function $\tau$, it is considered $e_{\tau, i}(t)=\tau(t)^{i}, \quad e_{\tau, i}^{x}(t)=(\tau(t)-\tau(x))^{i}$. Associated with the function $\tau$ the following differential operator is defined, see [13]

$$
D_{\tau}^{i} f(t):=D^{i}\left(f \circ \tau^{-1}\right)(\tau(t)) .
$$


We notice that $D_{\tau}^{0}=\mathbb{I}$. The previous definition is equivalent to this other:

$$
D^{0, \tau^{\prime}}=\mathbb{I}, \quad D^{1, \tau^{\prime}}=\frac{1}{\tau^{\prime}} D^{1}, \quad D^{i+1, \tau^{\prime}}=D^{1, \tau^{\prime}} \circ D^{i, \tau^{\prime}}, i \in \mathbb{N} .
$$

This differential operator has been dealt by the author inside approximation theory in [7] and [3].

It it easy to observe that for $x \in(0,1)$,

$$
D_{\tau}^{i} e_{\tau, j}=\left\{\begin{array}{ll}
\frac{j !}{(j-i) !} e_{\tau, j-i}, & \text { if } j \geq i ; \\
0, & \text { if } j<i,
\end{array} \quad \text { and } D_{\tau}^{i} e_{\tau, j}^{x}= \begin{cases}\frac{j !}{(j-i) !} e_{\tau, j-i}^{x}, & \text { if } j \geq i \\
0, & \text { if } j<i\end{cases}\right.
$$

\section{QUALITATIVE KOROVKIN TYPE RESULT}

Here is one of the main result of the paper, extension of the classical result of Korovkin.

Theorem 3.1. Let $L_{n}: C^{m}[0,1] \rightarrow C^{m}[0,1]$ be a sequence of linear operators fulfilling the following shape preserving property:

$$
\text { if } D_{\tau}^{m} f \geq 0 \text { then } D_{\tau}^{m} L_{n} f \geq 0
$$

Suppose we have three functions, $F_{0}, F_{1}, F_{2} \in C^{m}[0,1]$ such that $T=\left\{D_{\tau}^{m} F_{0}, D_{\tau}^{m} F_{1}, D_{\tau}^{m} F_{2}\right\}$ is a Tschebyshev System on $C[0,1]$, then the following sentences are equivalent:

(i) $\mathcal{L}\left(D_{\tau}^{m} L_{n} F_{i}(x)\right)=D_{\tau}^{m} F_{i}(x), \quad i=0,1,2$,

(ii) $\mathcal{L}\left(D_{\tau}^{m} L_{n} f(x)\right)=D_{\tau}^{m} f(x)$ for all function $f \in C^{m}[0,1]$.

\section{Proof.}

$(i i) \Rightarrow(i)$ is trivial. We are going to prove the converse. First of all, we consider that $x \in(0,1)$. We define the function $\phi \in C^{m}[0,1]$ as $\phi(t)=f(t)-\frac{D_{\tau}^{m} f(x)}{D_{\tau}^{m} G(x)} G(t)$, where $D_{\tau}^{m} G$ is a $T$-polynomial of $T=\left\{D_{\tau}^{m} F_{0}, D_{\tau}^{m} F_{1}, D_{\tau}^{m} F_{2}\right\}$, a Tchebychev System on $[0,1]$, with $D_{\tau}^{m} G(x) \neq 0$.

$D_{\tau}^{m} \phi$, is continuous and it vanishes at $x$, then for all positive real number $\epsilon$ there exists $\delta>0$ such that if $|t-x|<\delta$, then

$$
-\epsilon \leq D_{\tau}^{m} \phi(t) \leq \epsilon
$$

On the other hand $D_{\tau}^{m} \phi$ is bounded on $[0,1]$, then there exists $M>0$ such that

$$
-M \leq D_{\tau}^{m} \phi(t) \leq M .
$$

By property $\mathbf{P 2}$, for $x$, we can find two non negative $T$-polynomials, $f_{x}, h$, where the first function has a double root at $x$ and the second function is greater than or equal to 1 on $[0,1]$. Let $k=\min _{|t-x| \geq \delta} f_{x}(t)>0$ and $F_{x}, H \in C^{m}[0,1]$ such that $D_{\tau}^{m} F_{x}=f_{x}, D_{\tau}^{m} H=h$, then the following inequality is satisfied for $t \in[0,1]$

$$
-\epsilon D_{\tau}^{m} H(t)-\frac{M}{k} D_{\tau}^{m} F_{x}(t) \leq D_{\tau}^{m} \phi(t) \leq \epsilon D_{\tau}^{m} H(t)+\frac{M}{k} D_{\tau}^{m} F_{x}(t),
$$

or equivalently on $[0,1]$,

$$
D_{\tau}^{m}\left(-\epsilon H-\frac{M}{k} F_{x}\right) \leq D_{\tau}^{m} \phi \leq D_{\tau}^{m}\left(\epsilon H+\frac{M}{k} F_{x}\right) .
$$

Applying the shape preserving property (3.3), linearity and then evaluating at $x$ we have,

$$
-\epsilon D_{\tau}^{m} L_{n} H(x)-\frac{M}{k} D_{\tau}^{m} L_{n} F_{x}(x) \leq D_{\tau}^{m} L_{n} \phi(x) \leq \epsilon D_{\tau}^{m} L_{n} H(x)+\frac{M}{k} D_{\tau}^{m} L_{n} F_{x}(x) .
$$


Since $\epsilon$ is arbitrary, we can choose $\epsilon=\frac{1}{n}$. As $F_{x}, H$ belong to space spanned by $F_{0}, F_{1}, F_{2}$, then we use the hypothesis $(i)$ to get $\mathcal{L}\left(D_{\tau}^{m} L_{n} F_{x}(x)\right)=D_{\tau}^{m} F_{x}(x)=0$ and $\mathcal{L}\left(D_{\tau}^{m} L_{n} H(x)\right)=$ $D_{\tau}^{m} H(x)=h(x)$.

From (3.4) and property (III), we have that $\mathcal{L}\left(\epsilon D_{\tau}^{m} L_{n} H(x)+\frac{M}{k} D_{\tau}^{m} L_{n} F_{x}(x)\right)=0$. Then from property (IV), we deduce that $\mathcal{L}\left(D_{\tau}^{m} L_{n} \phi(x)\right)=0$ or equivalently $\mathcal{L}\left(D_{\tau}^{m} L_{n} f(x)-\right.$ $\left.\frac{D_{\tau}^{m} f(x)}{D_{\tau}^{m} G(x)} D_{\tau}^{m} L_{n} G(x)\right)=0$, so $\mathcal{L}\left(D_{\tau}^{m} L_{n} f(x)\right)=D_{\tau}^{m} f(x)$.

Now, we will prove the result for the end points of the interval $x=0$ and $x=1$. In this case, we define $\phi$ as $\phi(t)=f(t)-G(t)$, where $D_{\tau}^{m} G$ is a $T$-polynomial, with $D_{\tau}^{m} G(0)=$ $D_{\tau}^{m} f(0), D_{\tau}^{m} G(1)=D_{\tau}^{m} f(1)$.

Again we use the continuity, in this case in 0 and 1, and the bound $M$ of $D_{\tau}^{m} \phi$, as well as the fact that $D_{\tau}^{m} \phi$ vanishes at the endpoints of the interval. Then for all $\epsilon>0$, there exist $\delta>0$ such that for $0 \leq t \leq \delta, 1-\delta \leq t \leq 1$

$$
-\epsilon \leq D_{\tau}^{m} \phi(t) \leq \epsilon \text { and }-M \leq D_{\tau}^{m} \phi(t) \leq M .
$$

Now, we choose $F_{01} \in C^{m}[0,1]$, where $D_{\tau}^{m} F_{01}$ is a $T$-polynomial, $D_{\tau}^{m} F_{01}(0)=D_{\tau}^{m} F_{01}(1)=$ 0 and $D_{\tau}^{m} F_{01} \geq 0$. Now, we take $k=\min _{\delta \leq x \leq 1-\delta} D_{\tau}^{m} F_{01}(t)>0$. Then we have the following inequalities on $[0,1]$

$$
-\frac{M}{k} D_{\tau}^{m} F_{01}-\epsilon \leq D_{\tau}^{m} \phi \leq \epsilon+\frac{M}{k} D_{\tau}^{m} F_{01} .
$$

Finally, we can end the proof with similar arguments to the other case.

\section{ASYMPTOTIC CONDITION}

Once guaranteed the generalized convergence of the process, we are going to analyze the sequence $D_{\tau}^{m} L_{n} f(x)-D_{\tau}^{m} f(x)$ comparing it with another sequence of real numbers $\lambda_{n}$ with $\mathcal{L}\left(\lambda_{n}\right)=0$. The purpose is to obtain an asymptotic condition. Here it is the corresponding result. Again it is a Korovkin type result.

Theorem 4.2. Let $L_{n}$ be the sequence of linear operators as that of Section 3. Let $x \in(0,1)$ and let us assume that there exist a sequence $\lambda_{n}$ of positive real numbers, with $\mathcal{L}\left(\lambda_{n}\right)=0$ and three strictly positive functions $w_{0}, w_{1}$ and $w_{2}$ defined on $(0,1)$ with $w_{i} \in C^{2-i}(0,1)$ such that, for $s \in\{m, m+$ $1, m+2, m+4\}$,

$$
\mathcal{L}\left(\frac{D_{\tau}^{m} L_{n} e_{\tau, s}^{x}(x)-D_{\tau}^{m} e_{\tau, s}^{x}(x)}{\lambda_{n}}\right)=w_{2}^{-1} D^{1}\left(w_{1}^{-1} D^{1}\left(w_{0}^{-1} D_{\tau}^{m} e_{\tau, s}^{x}\right)\right)(x)
$$

Then, for $f \in C^{m}(0,1), m+2$ times differentiable in some neighborhood of $x$,

$$
\mathcal{L}\left(\frac{D_{\tau}^{m} L_{n} f(x)-D_{\tau}^{m} f(x)}{\lambda_{n}}\right)=w_{2}^{-1} D^{1}\left(w_{1}^{-1} D^{1}\left(w_{0}^{-1} D_{\tau}^{m} f\right)\right)(x) .
$$

\section{Proof.}

The proof similar to the one we can find in [3], with the proper changes. First of all, we apply the Taylors's formula to the function $D_{\tau}^{m} f \circ \tau^{-1}$ centered at a point $\tau(x)$ and evaluated at $\tau(t), t \in(0,1)$, i.e.:

$$
D_{\tau}^{m} f \circ \tau^{-1}(\tau(t))=\sum_{s=0}^{2} \frac{1}{s !} D^{s}\left(D_{\tau}^{m} f \circ \tau^{-1}\right)(\tau(x))(\tau(t)-\tau(x))^{s}+h(\tau(t)-\tau(x))(\tau(t)-\tau(x))^{2},
$$

where $h$ is a continuous function that vanishes at zero. Now using the definition of the differential operator (2.1) and the notation of Section 2, we have: 


$$
\begin{gathered}
D_{\tau}^{m} f(t)=D_{\tau}^{0}\left(D_{\tau}^{m} f\right)(x) e_{\tau, 0}^{x}(t)+D_{\tau}^{1}\left(D_{\tau}^{m} f\right)(x) e_{\tau, 1}^{x}(t) \\
+\frac{1}{2} D_{\tau}^{2}\left(D_{\tau}^{m} f\right)(x) e_{\tau, 2}^{x}(t)+h(\tau(t)-\tau(x)) e_{\tau, 2}^{x}(t) .
\end{gathered}
$$

Using (2.2), we can write

$$
D_{\tau}^{m} f(t)=D_{\tau}^{m}\left(\sum_{s=0}^{2} \frac{1}{(m+s) !} D_{\tau}^{s}\left(D_{\tau}^{m} f\right)(x) e_{\tau, m+s}^{x}+H_{x}\right)(t)
$$

with $H_{x} \in C^{m}(J)$ and $D_{\tau}^{m} H_{x}(t)=h(\tau(t)-\tau(x)) e_{\tau, 2}^{x}(t)$. Then, we apply the linear operator and evaluate at $x$ to obtain

$$
D_{\tau}^{m} L_{n} f(x)=D_{\tau}^{m} L_{n}\left(\sum_{s=0}^{2} \frac{1}{(m+s) !} D_{\tau}^{s}\left(D_{\tau}^{m} f\right)(x) e_{\tau, m+s}^{x}+H_{x}\right)(x)
$$

By linearity,

$$
D_{\tau}^{m} L_{n} f(x)=\sum_{s=0}^{2} \frac{1}{(m+s) !} D_{\tau}^{s}\left(D_{\tau}^{m} f\right)(x) D_{\tau}^{m} L_{n} e_{\tau, m+s}^{x}(x)+D_{\tau}^{m} L_{n} H_{x}(x) .
$$

Introducing this term, $D_{\tau}^{m} f(x)=\sum_{s=0}^{2} \frac{1}{(m+s) !} D_{\tau}^{s}\left(D_{\tau}^{m} f\right)(x) D_{\tau}^{m} e_{\tau, m+s}^{x}(x)$, to both sides of the equality and dividing by $\lambda_{n}$

$$
\begin{gathered}
\frac{D_{\tau}^{m} L_{n} f(x)-D_{\tau}^{m} f(x)}{\lambda_{n}}=\sum_{s=0}^{2} \frac{1}{(m+s) !} D_{\tau}^{s}\left(D_{\tau}^{m} f\right)(x) \frac{D_{\tau}^{m} L_{n} e_{\tau, m+s}^{x}(x)-D_{\tau}^{m} e_{\tau, m+s}^{x}(x)}{\lambda_{n}}+ \\
\frac{D_{\tau}^{m} L_{n} H_{x}(x)}{\lambda_{n}} .
\end{gathered}
$$

Now, we consider the hypothesis (4.5) for $m=0, m=1, m=2$. After some calculations,

$$
\begin{gathered}
\mathcal{L}\left(\sum_{s=0}^{2} \frac{1}{(m+s) !} D_{\tau}^{s}\left(D_{\tau}^{m} f\right)(x) \frac{D_{\tau}^{m} L_{n} e_{\tau, m+s}^{x}(x)-D_{\tau}^{m} e_{\tau, m+s}^{x}(x)}{\lambda_{n}}\right)= \\
w_{2}^{-1} D^{1}\left(w_{1}^{-1} D^{1}\left(w_{0}^{-1} D_{\tau}^{m} f\right)\right)(x) .
\end{gathered}
$$

Finally, the proof of 4.6 will be finished if we prove that $\mathcal{L}\left(\frac{D_{\tau}^{m} L_{n} H_{x}(x)}{\lambda_{n}}\right)=0$ and the proof will be finished.

To do this, we use continuity arguments on the function $h$ to guarantee the existence of a neighborhood of $x$, say $\theta_{x}$, for a given $\epsilon>0$, such that for $t \in \theta_{x}$,

$$
|h(\tau(t)-\tau(x))|<\epsilon .
$$

Then, for all $t \in[0,1]$,

$$
\left|D_{\tau}^{m} H_{x}(t)\right|=|h(\tau(t)-\tau(x))| e_{\tau, 2}^{x}(t) \leq \epsilon e_{\tau, 2}^{x}(t)+\max \{0,|h(\tau(t)-\tau(x))|-\epsilon\} e_{\tau, 2}^{x}(t) .
$$

Let us consider a function $W \in C^{m}[0,1]$ such that $D_{\tau}^{m} W(t)=\max \{0,|h(\tau(t)-\tau(x))|-\epsilon\} e_{\tau, 2}^{x}(t)$. As $D_{\tau}^{m} W$ vanishes in $\theta_{x}$, then, for a sufficiently large constant $M$, one has $\left|D_{\tau}^{m} W(t)\right| \leq$ $M D_{\tau}^{m} e_{\tau, m+4}^{x}(t)$. So, gathering the last inequalities we get,

$$
\left|D_{\tau}^{m} H_{x}(t)\right| \leq \frac{2 \epsilon}{(m+2) !} D_{\tau}^{m} e_{\tau, m+2}^{x}(t)+M D_{\tau}^{m} e_{\tau, m+4}^{x}(t)
$$


We use the shape preserving property (3.3), and divide by $\lambda_{n}>0$, to obtain, after evaluating at the point $x$,

$$
\left|\frac{D_{\tau}^{m} L_{n} H_{x}(x)}{\lambda_{n}}\right| \leq \frac{2 \epsilon}{(m+2) !} \frac{D_{\tau}^{m} L_{n} e_{\tau, m+2}^{x}(x)}{\lambda_{n}}+M \frac{D_{\tau}^{m} L_{n} e_{\tau, m+4}^{x}(x)}{\lambda_{n}} .
$$

As regards the hypothesis of the result for $s=m+2$ and $s=m+4$, after some calculations using (2.2), we can write respectively,

$$
\mathcal{L}\left(\frac{D_{\tau}^{m} L_{n} e_{\tau, m+2}^{x}(x)}{\lambda_{n}}\right)=\frac{2 \tau^{\prime}(x)^{2}}{w_{2}(x) w_{1}(x) w_{0}(x)}>0
$$

and

$$
\mathcal{L}\left(\frac{D_{\tau}^{m} L_{n} e_{\tau, m+4}^{x}(x)}{\lambda_{n}}\right)=0 .
$$

Finally, properties (III) and (IV) and the fact that $\epsilon>0$ was arbitrary, allow us to finish the proof.

\section{FURTHER RESULTS}

From now on, we will assume that the sequence of linear operators is endowed with an asymptotic condition of the type (4.6). We are going to deduce some consequences of the latter fact. First of all, the existence of an asymptotic condition allows us to establish a local version of the shape preserving property. We use the notation $a_{n}=o_{\mathcal{L}}\left(b_{n}\right)$ to refer to two sequences such that $a_{n}, b_{n} \in \mathcal{S}_{0}, \mathcal{L}\left(a_{n}\right)=\mathcal{L}\left(b_{n}\right)=\mathcal{L}\left(\frac{a_{n}}{b_{n}}\right)=0$.

Lemma 5.1. Let $h \in C^{m}[0,1]$ and $x \in(0,1)$. We assume that there exists a neighborhood $N_{x}$ of $x$ where $D_{\tau}^{m} h \geq 0$. Then,

$$
D_{\tau}^{m} L_{n} h(x) \geq 0+o_{\mathcal{L}}\left(\lambda_{n}\right) .
$$

Proof. Let $x_{1}, x_{2} \in N_{x}$ with $x_{1}<x<x_{2}$ and let $\tau_{1}, \tau_{2}$ belong to the space spanned by $1, \tau^{1}, \ldots, \tau^{m}$ such that for $j=1,2$ and $0 \leq i \leq m, D_{\tau}^{i} \tau_{j}\left(x_{j}\right)=D_{\tau}^{i} h\left(x_{j}\right)$ (notice that $\left\{1, \tau^{1}, \ldots, \tau^{m}\right\}$ is a Tchebychev system). Let $\widetilde{h} \in C^{m}[0,1]$ be defined as:

$$
\widetilde{h}(t)= \begin{cases}\tau_{1}(t) & t<x_{1} \\ h(t) & x_{1} \leq t \leq x_{2} \\ \tau_{2}(t) & x_{2}<t\end{cases}
$$

Then, on $[0,1], D_{\tau}^{m} \widetilde{h} \geq 0$ and on $\left(x_{1}, x_{2}\right), D_{\tau}^{m}(\widetilde{h}-h)=0$. Indeed, it is enough to recall that for $i=0,1, \ldots, m-1, D_{\tau}^{m} \tau^{i}=0$ and observe that $D_{\tau}^{m} \tau^{m}=m$ !. Finally, using the existence of an asymptotic condition (4.6), yields $D_{\tau}^{m} L_{n} \widetilde{h}(x)-D_{\tau}^{m} L_{n} h(x)=o_{\mathcal{L}}\left(\lambda_{n}\right)$, and from (3.3)

$$
0 \leq D_{\tau}^{m} L_{n} \widetilde{h}(x)=D_{\tau}^{m} L_{n} h(x)+o_{\mathcal{L}}\left(\lambda_{n}\right) .
$$

If $g \in C^{m}[0,1]$ is a solution on $(a, b) \subset[0,1]$ of the ordinary differential equation

$$
w_{2}^{-1} D^{1}\left(w_{1}^{-1} D^{1}\left(w_{0}^{-1} D_{\tau}^{m} y\right) \equiv 0,\right.
$$

by asymptotic condition (4.6) it is obvious that if $x \in(a, b), D_{\tau}^{m} L_{n} f(x)-D_{\tau}^{m} f(x)=o_{\mathcal{L}}\left(\lambda_{n}\right)$, but the converse is also true, as we can see in the next result.

Theorem 5.3. Let $a, b \in(0,1)$ with $a<b$. If $f \in C^{m}[0,1]$ satisfies $D_{\tau}^{m} L_{n} f(x)-D_{\tau}^{m} f(x)=o_{\mathcal{L}}\left(\lambda_{n}\right)$ at each point $x \in(a, b)$, then $f$ is a solution of (5.7). 
Before the proof, we write some remarks. The ordinary differential equation (5.7) is of order $m+2$, with fundamental set of solutions $\left\{1, \tau, \ldots, \tau^{m-1}, y_{1}, y_{2}\right\}$. The change of variable $z=$ $D_{\tau}^{m} v$ makes equation (5.7) become the following one of second order:

$$
\frac{1}{w_{2}} D^{1}\left(\frac{1}{w_{1}} D^{1}\left(\frac{z}{w_{0}}\right)\right) \equiv 0 .
$$

The following lemma, whose proof can be found in, [7, Lemma 1] it is necessary for the proof of the theorem.

Lemma 5.2. Let $J$ be a bounded open subinterval of $[0,1]$. Let $g, h \in C(J)$ and $t_{0}, t_{1}, t_{2} \in J$ such that $t_{0} \in\left(t_{1}, t_{2}\right), g\left(t_{1}\right)=g\left(t_{2}\right)=0$ and $g\left(t_{0}\right)>0$. Then there exist a real number $\alpha<0$, a solution of the differential equation (5.8) on $J$, say $z$, and a point $x \in\left(t_{1}, t_{2}\right)$ such that for all $t \in\left[t_{1}, t_{2}\right]$, $\alpha h(t)+z(t) \geq g$, and at the point $x, \alpha h(x)+z(x)=g(x)$.

Let us proceed to the proof of Theorem 5.3. Let $f \in C^{m}[0,1]$ and let $z_{0}$ be the unique solution of (5.8) such that $z_{f}(a)=D_{\tau}^{m} f(a)$ and $z_{0}(b)=D_{\tau}^{m} f(b)$ and suppose that there exists $x_{0} \in$ $(a, b), z_{f}\left(x_{0}\right)>D_{\tau}^{m} f\left(x_{0}\right)$ (by linearity, one may proceed analogously if the other inequality is assumed). We apply Lemma 5.2 with $g=z_{f}-D_{\tau}^{m} f, h=D_{\tau}^{m} e_{\tau, m+2}^{x}, t_{1}=a, t_{2}=b, t_{0}=x_{0}$. In this case, there exist $\alpha<0, z$ solution of (5.8) and $x \in(a, b)$ such that,

$$
\alpha D_{\tau}^{m} e_{\tau, m+2}^{x}(t)+z(t) \geq z_{f}(t)-D_{\tau}^{m} f(t), t \in(a, b),
$$

$$
\alpha D_{\tau}^{m} e_{\tau, m+2}^{x}(x)+z(x)=z_{f}(x)-D_{\tau}^{m} f(x) .
$$

Now if we consider $Z_{f} \in C^{m}[0,1], D_{\tau}^{m} Z_{f}=z_{f}$ and $Z \in C^{m}[0,1], D_{\tau}^{m} Z=z$, applying the localization Lemma 5.1 and dividing by $\lambda_{n}$, from (5.9) we obtain,

$$
\begin{gathered}
\alpha \frac{D_{\tau}^{m} L_{n} e_{\tau, m+2}^{x}(x)-D_{\tau}^{m} e_{\tau, m+2}^{x}(x)}{\lambda_{n}}+\frac{D_{\tau}^{m} L_{n} Z(x)-D_{\tau}^{m} Z(x)}{\lambda_{n}} \geq \\
\frac{D_{\tau}^{m} L_{n} Z_{f}(x)-D_{\tau}^{m} Z_{f}(x)}{\lambda_{n}}+\frac{o_{\mathcal{L}}\left(\lambda_{n}\right)}{\lambda_{n}} .
\end{gathered}
$$

We use property (II) to get

$$
\begin{gathered}
\alpha \mathcal{L}\left(\frac{D_{\tau}^{m} L_{n} e_{\tau, m+2}^{x}(x)-D_{\tau}^{m} e_{\tau, m+2}^{x}(x)}{\lambda_{n}}\right)+\mathcal{L}\left(\frac{D_{\tau}^{m} L_{n} Z(x)-D_{\tau}^{m} Z(x)}{\lambda_{n}}\right) \geq \\
\mathcal{L}\left(\frac{D_{\tau}^{m} L_{n} Z_{f}(x)-D_{\tau}^{m} Z_{f}(x)}{\lambda_{n}}\right),
\end{gathered}
$$

and finally we apply asymptotic condition (4.6) to obtain the following expression in contradiction with the hypothesis,

$$
\alpha \frac{2 \tau^{\prime}(x)^{2}}{w_{2}(x) w_{1}(x) w_{0}(x)}>0,
$$

to conclude that $D_{\tau}^{m} f$ is a solution of (5.8), so $f$ is a solution of (5.7).

\section{AN EXAMPLE}

As it was pointed out in the introductory section, in this section we apply the results of the paper to the notion of generalized convergence considered by V. Loku and N. L. Braha [12]. Let $p_{n}$ be a non negative, non increasing real sequence. Let $N_{n}^{p} C_{n}^{1}(\cdot)$ be the linear transformation that assigns to each real sequence $x_{n}$ this other

$$
N_{n}^{p} C_{n}^{1}\left(x_{n}\right)=\frac{1}{\sum_{k=1}^{n} p_{k}} \sum_{k=1}^{n} p_{k} \frac{1}{k} \sum_{v=1}^{k} x_{v}, \quad n \in \mathbb{N} .
$$


The sequence $x_{n}$ is said to be Nörlund-Cesáro summable by the weighted mean determined by $p_{n}$, or briefly $\left(N, p_{n}\right)(C, 1)$-summable if

$$
\lim N_{n}^{p} C_{n}^{1}\left(x_{n}\right)=\ell .
$$

In that case, the following notation is used: $N_{n}^{p} C_{n}^{1}-\lim x_{n}=\ell$. Moreover, the set of all $\left(N, p_{n}\right)(C, 1)$-summable sequences is denoted by $N_{n}^{p} C_{n}^{1}$.

Let us now recover the sequence of operators $L_{n} f(t)=\left(1+a_{n}\right) B_{n}^{\tau} f(t)$ with $a_{n} \in N_{n}^{p} C_{n}^{1}$ and $a_{n} \geq 1$. In order to prove the following statement, no Korovkin-type proof is needed.

Theorem 6.4. Let $F_{0}, F_{1}, F_{2} \in C^{m}[0,1]$ such that $\left\{D_{\tau}^{m} F_{0}, D_{\tau}^{m} F_{1}, D_{\tau}^{m} F_{2}\right\}$ is a Tschebyshev System on $C[0,1]$. Then the followings sentences are equivalent:

(i) $N_{n}^{p} C_{n}^{1}-\lim D_{\tau}^{m} L_{n} F_{i}(x)=D_{\tau}^{m} F_{i}(x), i=0,1,2$

(ii) $N_{n}^{p} C_{n}^{1}-\lim D_{\tau}^{m} L_{n} f(x)=D_{\tau}^{m} f(x), i=0,1,2$, for all function $f \in C^{m}[0,1]$.

Following the results of the paper, for the proof of the theorem we only have to check that the shape preserving property (3.3) is fulfilled, and that the linear functional $\mathcal{L}\left(x_{n}\right)=N_{n}^{p} C_{n}^{1}-$ $\lim x_{n}$, defined on $\mathcal{S}_{0}=N_{n}^{p} C_{n}^{1}$ satisfies properties (I)-(VI). Moreover, all the results of the paper apply to this situation accordingly.

Finally, for the sake of completeness, I write a remark about a recent paper. In [15], the authors defined a new sequence of linear operators and proved a result under statistical convergence. The main theorem of the current paper shows an alternative approach to the problem.

\section{REFERENCES}

[1] T. Acar and S. A. Mohiuddine, Statistical $(C, 1)(E, 1)$ Summability and Korovkin's Theorem, Filomat, 30:02, 2016, 387-393.

[2] D. Cárdenas-Morales, P. Garrancho and I. Raşa, Bernstein-type operators which preserve polynomials, Comput. Math. Appl., 62, 2011, 158-163.

[3] D. Cárdenas-Morales and P. Garrancho, B-statistical A-summability in conservative approximation, Math. Inequal. Appl.,19(3), 2016, 923-936.

[4] O. Duman, M. K. Khan and C. Orhan, A-Statistical convergence of approximating operators, Math. Inequal. Appl. 6, 2003, 689-699.

[5] H. Fast, Sur la convergence statistique, Colloq. Math., 2, 1951, 241-244.

[6] A. D. Gadjiev and C. Orhan, Some approximation theorems via statistical convergence, Rocky Mountain J. Math., 32 , 2002, 129-138.

[7] P. Garrancho and D. Cárdenas-Morales, A converse of asymptotic formulae in simultaneous approximation, Appl. Math. and Comp., 217, 2010, 2676-2683.

[8] A. Karaisa, Statistical $\alpha \beta$-Summability and Korovkin Type Approximation Theorem, Filomat ,30:13, $2016,3483-3491$.

[9] V. Karakaya and A. Karaisa, Korovkin type approximation theorems for weighted $\alpha \beta-$ statistical convergence, Bull. Math. Sci., 5, 2015, 159-169.

[10] J. P. King and J. J. Swetits, Positive linear operators and summability, Austral J. Math., 11, 1970, $281-291$.

[11] P. P. Korovkin, Linear operators and approximation theory, Hindustan Publishing Corp., Delhi, India, 1960.

[12] V. Loku and N. L. Braha, Tauberian Theorems by Weighted Summability Method, Armenian J. of Math., 9(1), 2017, 35-42.

[13] A.-J. López-Moreno and F.-J. Muñoz-Delgado, Asymptotic expansion of multivariate conservative linear operators, J. Comput. Appl. Math, 150, 2003, 219-251.

[14] G. G. Lorentz, A contribution to the theory of divergent sequences, Acta. Math., 80, 1948, 167-190.

[15] H. Sharma, R. Maurya and C. Gupta, Approximation properties of Kantorovich Type Modifications of p, q-Meyer-KönigZeller Operators, Constr. Math. Anal., 1(1), 2018, 58-72.

UNIVERSITY OF JAÉN,

DEPARTMENT OF MATHEMATICS,

PARAJE DE LAS LAGUNILLAS, 23071,

JAÉN, SPAIN

E-mail address: pgarran@u jaen.es 